\title{
Decision making in multiobjective optimization problems under uncertainty: balancing between robustness and quality
}

\section{Yue Zhou-Kangas $^{1}$ (D) Kaisa Miettinen ${ }^{1}$}

Received: 29 April 2018 / Accepted: 3 November 2018 / Published online: 16 November 2018 (c) The Author(s) 2018

\begin{abstract}
As an emerging research field, multiobjective robust optimization employs minmax robustness as the most commonly used concept. Light robustness is a concept in which a parameter, tolerable degradations, can be used to control the loss in the objective function values in the most typical scenario for gaining in robustness. In this paper, we develop a lightly robust interactive multiobjective optimization method, LiRoMo, to support a decision maker to find a most preferred lightly robust efficient solution with a good balance between robustness and the objective function values in the most typical scenario. In LiRoMo, we formulate a lightly robust subproblem utilizing an achievement scalarizing function which involves a reference point specified by the decision maker. With this subproblem, we compute lightly robust efficient solutions with respect to the decision maker's preferences. With LiRoMo, we support the decision maker in understanding the lightly robust efficient solutions with an augmented value path visualization. We use two measures 'price to be paid for robustness' and 'gain in robustness' to support the decision maker in considering the trade-offs between robustness and quality. As an example to illustrate the advantages of the method, we formulate and solve a simple investment portfolio optimization problem.
\end{abstract}

Keywords Multiobjective robust optimization - Interactive methods · Light robust efficiency · Handling uncertainty · Trade-off between robustness and quality ·

Decision support

Yue Zhou-Kangas

yue.y.zhou-kangas@jyu.fi

Kaisa Miettinen

kaisa.miettinen@jyu.fi

1 University of Jyväskylä, Faculty of Information Technology, P.O. Box 35 (Agora), 40014 University of Jyväskylä, Finland 


\section{Introduction}

Many decision-making problems involve multiple criteria to be optimized and they also include uncertainty from different sources such as uncertain future developments and imprecise measurements. Due to the uncertainty, the outcome of implementing a decision can become unexpected and undesired. In recent years, both researchers and practitioners have started to pay attention to dealing with multiple criteria and the involved uncertainty simultaneously. The approaches employed depend on the availability of data and the expert knowledge of the decision maker. When enough data about the uncertainty are available, problems can be solved with stochastic approaches (see e.g., Gutjahr and Pichler 2016) and when the expert judgments on fuzzy memberships can be relied on, fuzzy approaches can be implemented (see e.g., Inuiguchi et al. 1990). On the other hand, when there is no sufficient data available or the decision maker does not have sufficient knowledge to judge the memberships, robust optimization approaches can be utilized (see e.g., Ide and Schöbel 2016; Wiecek and Dranichak 2016).

In robust optimization approaches, typically the uncertainty is modeled as parameters whose exact values are not known but are assumed to stem from a set. We call this set an uncertainty set. Possible realizations of the unknown parameters are called scenarios, which are the elements in the uncertainty set. We call the most typical or expected scenario the nominal scenario and the objective function values in the nominal scenario as the nominal quality. In order to find solutions when uncertainty is taken into account, different robustness concepts have been developed. Flimsily robust solutions (Bitran 1980; Ide and Schöbel 2016) are the best solutions in one of the possible realizations of the uncertain parameters. Highly robust solutions (Bitran 1980; Dranichak and Wiecek 2018; Goberna et al. 2018; Ide and Schöbel 2016) are the best solutions in all the possible realizations of uncertain parameters at the same time. The most common concepts used in multiobjective robust optimization belong to the family of minmax robustness concepts (e.g., Bokrantz and Fredriksson 2017; Ehrgott et al. 2014; Eichfelder et al. 2017; Kuroiwa and Lee 2012). For minmax robustness, we optimize the objective functions in the worst case over all scenarios. The solutions computed are said to be minmax robust efficient.

However, minmax robust efficient solutions are not always easy to compute. In addition, the conservatism of minmax robustness has been recognized in single objective cases (see e.g., Ben-Tal et al. 2010; Bertsimas and Sim 2004; Schöbel 2014), i.e., the objective function value of a minmax robust solution is usually not that good in other scenarios. Also, the decision maker may not be willing to make decisions based on the worst possible realizations of the uncertain parameters. On the other hand, if a solution is found only by optimizing in the nominal scenario, the possibility of realizations of other scenarios is ignored. So, the decision maker may want to focus on the nominal scenario but make decisions which are still valid in other scenarios. Based on the consideration of both robustness and the nominal quality, developments in single objective cases have led to different concepts in controlling the degradation of the objective function value in the nominal scenario (see e.g., Ben-Tal et al. 2004, 2009, 2010; Bertsimas and Sim 2004; Goerigk and Schöbel 2016; Schöbel 2014). These concepts share the same idea of balancing between robustness and the nominal quality. 
Similar thoughts have initiated developments in multiobjective optimization in Hassanzadeh et al. (2014), Ide and Schöbel (2016), Kuhn et al. (2016). In Hassanzadeh et al. (2014), a multiobjective version of the concept proposed in Bertsimas and Sim (2004) has been developed and the robust optimization problem is solved based on the augmented Chebychev function (see e.g., Steuer and Choo 1983). The concept of light robustness has been originally proposed in Fischetti and Monaci (2009) for single objective linear problems with uncertain parameters which stem from an interval uncertainty set and generalized in Schöbel (2014) for other types of uncertainty sets and quasi-convex objective functions. It has been extended to light robust efficiency for multiobjective cases in Ide and Schöbel (2016). The idea of light robust efficiency is to find a robust solution with tolerable degradations in the nominal quality. Concepts based on light robustness have been developed in Kuhn et al. (2016) for problems where uncertain parameters are involved in one of the two objectives in bi-objective optimization problems. An algorithm for bi-objective combinatorial optimization problems with a finite uncertainty set has also been developed. Solution methods for supporting a decision maker to find a most preferred lightly robust efficient solution when uncertainty is involved in multiple objectives have not been published.

In light robustness, the loss in the nominal quality is controlled by a parameter, indicating tolerable degradations in nominal quality. With respect to the tolerable degradations in the nominal quality, we optimize in the worst case to seek for a most robust solution. By doing so, usually, the computed solutions do not have as good quality in the worst case as minmax robust efficient solutions but they are usually better in terms of nominal quality. It is proven in Ide and Schöbel (2016) that with a sufficiently large tolerance on the degradations in the nominal quality, a computed lightly robust efficient solution is a minmax robust efficient solution. The relationships between minmax robust efficient, lightly robust efficient and nominal efficient solutions are analyzed in Zhou-Kangas and Schöbel (2018). It is also proven that the lightly robust efficient solutions are good trade-offs between robustness and the nominal quality. Thus, with the concept of light robust efficiency, the decision maker can focus on the nominal quality but find solutions that are also valid in other scenarios. This is why we focus on utilizing the concept of light robustness.

In the solution process of a multiobjective optimization problem, the decision maker can utilize the parameter, tolerable degradations in the nominal quality, to control the trade-off between robustness and the nominal quality in order to find a solution with a good balance in both respects. As there usually is a set of efficient solutions, the decision maker needs support to understand the trade-offs among the objectives to choose a final solution. When robustness is considered in the solution process, the decision maker needs further support, not only for the trade-offs among the objectives but also the trade-offs between robustness and the nominal quality.

In this paper, we focus on applying light robustness for decision support and develop an interactive method, LiRoMo, to support the decision maker to find a good balance between robustness and the nominal quality by finding a most preferred lightly robust efficient solution. Interactive methods (see e.g., Miettinen 1999; Steuer 1986) are a category of solution methods for multiobjective optimization problems. With interactive methods, the interactive solution process starts by presenting an initial solution to the decision maker. If the decision maker is not satisfied, (s)he can specify pref- 
erences for a more desired solution. Then, a scalarized subproblem is solved to find a new solution which satisfies the preferences as well as possible. This process continues until the decision maker finds a most preferred solution. During the interactive solution process, the decision maker can learn about the problem, for example, some specific properties of the problem. The decision maker can also learn about the attainable objective function values and at the same time learn how achievable her or his own preferences are.

When seeking a most preferred lightly robust efficient solution, the decision maker needs to consider the robustness and the nominal quality of solutions at the same time. So, (s)he should be provided the opportunity not only to learn about the problem, the attainable objective function values, and her or his own preferences but also to learn about the trade-offs between robustness and the nominal quality. With an interactive method, we can provide such support. As a result, the decision maker can eventually find a final solution with a satisfactory balance between robustness and the nominal quality.

The decision maker directs the interactive solution process and we need to generate solutions utilizing the decision maker's preferences. As a common approach to compute efficient solutions in multiobjective optimization, scalarization functions combine multiple objectives (and preferences) into a single objective such that an optimal solution of the scalarized problem is an efficient solution to the multiobjective optimization problem. Scalarization for computing minmax robust efficient solutions has been discussed in Bokrantz and Fredriksson (2017). In the LiRoMo method, we formulate a lightly robust subproblem based on an achievement scalarization function (Wierzbicki 1986). For computing lightly robust efficient solutions efficiently, we reformulate the subproblem by utilizing the properties of the objective functions and the uncertainty sets. For supporting the decision maker to understand the trade-offs between robustness and the nominal quality, we quantify the gain in robustness and the price to be paid for robustness. In order to effectively illustrate the solutions to the decision maker, we augment the value path visualization (Miettinen 2014) to support the decision maker.

The rest of the paper is organized as follows: Sect. 2 describes the definitions and background information needed which is followed by Sect. 3 where we formulate the lightly robust subproblem based on the achievement scalarizing function and present its reformulation. In Sect. 4, we describe the LiRoMo method for supporting the decisionmaking process of balancing between robustness and quality. Then we formulate and solve an investment portfolio optimization problem to demonstrate the application of the LiRoMo method in Sect. 5 and conclude in Sect. 6.

\section{Preliminaries}

\subsection{Multiobjective optimization under uncertainty}

We consider multiobjective optimization problems where some parameters in the objective functions are unknown but stem from an uncertainty set $\mathcal{U}$ in the following form: 


$$
\left(\begin{array}{l}
\operatorname{minimize}\left(f_{1}(x, \xi), \ldots, f_{k}(x, \xi)\right) \\
\text { subject to } x \in X
\end{array}\right)_{\xi \in \mathcal{U}}
$$

where $x=\left(x_{1}, \ldots, x_{n}\right)^{T}$ is the decision vector which belongs to a feasible set $X \in \mathbb{R}^{n}$ and $\xi$ represents the uncertain parameters which stem from the set $\mathcal{U}$. We assume that a nominal scenario $\hat{\xi}$ is known. When robustness does not play a role, the problem is solved in the nominal scenario as a deterministic problem:

$$
\begin{array}{ll}
\operatorname{minimize} & \left(f_{1}(x, \hat{\xi}), \ldots, f_{k}(x, \hat{\xi})\right) \\
\text { subject to } & x \in X
\end{array}
$$

We call problem (2) a nominal problem and for each $x \in X$, we define the objective vector $f^{\text {nom }}(x):=\left(f_{1}(x, \hat{\xi}), \ldots, f_{k}(x, \hat{\xi})\right)^{T}$ as its nominal quality. We say that $x^{*}$ is an efficient (or Pareto optimal) solution to (2), if there does not exist any $x^{\prime} \in X$ such that $f_{i}^{\text {nom }}\left(x^{\prime}\right) \leq f_{i}^{\text {nom }}\left(x^{*}\right)$ for all $i=1, \ldots, k$ and $f_{j}^{\text {nom }}\left(x^{\prime}\right)<f_{j}^{\text {nom }}\left(x^{*}\right)$ for at least one $j$. The set of efficient solutions to (2) is denoted by $X^{\text {nom }}$. Problem (2) can be solved with methods for deterministic multiobjective optimization problems in the following form where no uncertainty is involved:

$$
\begin{array}{ll}
\operatorname{minimize} & \left(f_{1}(x), \ldots, f_{k}(x)\right) \\
\text { subject to } & x \in X
\end{array}
$$

For decision making, it is usually useful for the decision maker to know the ranges of the objective function values in $X^{\text {nom }}$. The ideal objective vector $z^{\text {ideal }}$ and the nadir objective vector $z^{\text {nad }}$ can provide information on the ranges. The ideal objective vector is formed by the individual optima of each objective function. The nadir objective vector gives the upper bounds of the objective vectors correspond to $X^{\text {nom }}$. The nadir objective vector can be approximated by for example the payoff table (see e.g., Miettinen 1999; Steuer 1986). This approximated vector can over or underestimate the nadir objective vector. There are also other ways to approximate the nadir objective vector (see e.g., Deb et al. 2010). For computational reasons, we also define a utopian objective vector $z^{\text {uto }}=\left(z_{1}^{\text {ideal }}-a, \ldots, z_{k}^{\text {ideal }}-a\right)^{T}$, where $a>0$ is a small scalar.

\subsection{Minmax robustness}

Minmax robust efficient solutions are found by optimizing in the worst case. The values of the uncertain parameters with which a solution $x \in X$ attains its worst objective function values are called the worst-case scenarios or simply worst cases. For a fixed $x \in X$, finding its worst-case objective vector(s) requires maximizing $k$ objectives simultaneously. If the problem is objective-wise uncertain, i.e., the uncertain parameters in the objective functions do not relate to each other, see (Ehrgott et al. 2014) for a formal definition, there exists a single worst-case scenario. Otherwise, there usually exists a set of worst-case scenarios.

In this paper, we employ the concept called point-based minmax robustness (see Fliege and Werner 2014; Kuroiwa and Lee 2012) for optimizing in the worst case. A 
point-based minmax robust optimization problem is defined as follows:

$$
\begin{aligned}
& \operatorname{minimize}\left(\sup _{\xi \in \mathcal{U}} f_{1}(x, \xi), \ldots, \sup _{\xi \in \mathcal{U}} f_{k}(x, \xi)\right) \\
& \text { subject to } x \in X .
\end{aligned}
$$

In this formulation, with a fixed $x \in X$, the worst-case objective function values are represented by an objective vector. This vector is formed by the individual maxima of each objective function with respect to the uncertain parameters involved. So, we consider a single worst case objective vector in the solution process of problems regardless of if the problem is objective-wise uncertain or not. However, the pointbased worst case usually does not realize i.e., the objective functions do not attain their worst values simultaneously unless the problem is objective-wise uncertain. We use $f^{\text {wc }}(x):=\left(f_{1}(x, \bar{\xi}), \ldots, f_{k}(x, \bar{\xi})\right)^{T}$ to represent a point-based minmax robust objective vector for a fixed $x \in X$. The vector $f^{\text {wc }}$ consists of the individual maxima of each objective function. We refer to $f^{\mathrm{wc}}$ as the worst case.

If the decision maker wishes to concentrate only on robustness, we can solve problem (4) to find point-based minmax robust efficient solutions for her or him. For helping the decision maker to understand the ranges of the objective function values of the point-based robust efficient solutions, we can identify the robust ideal objective vector $z^{\text {ideal, wc }}$ and the robust nadir objective vector $z^{\text {nad,wc }}$. The robust ideal objective vector $z^{\text {ideal,wc }}$ is formed by the individual minima of the objective functions in problem (4) and $z^{\text {nad,wc }}$ can be approximated by the payoff table. Corresponding to the nominal utopian objective vector, we also have the robust utopian objective vectors $z^{\text {uto,wc }}=\left(z_{1}^{\text {ideal,wc }}-a, \ldots, z_{k}^{\text {ideal,wc }}-a\right)^{T}$, where $a>0$ is a small scalar.

\subsection{Light robustness}

In the concept of light robustness, we assume that the set $X^{\text {nom }}$ is nonempty. The idea is that we first determine some $\hat{x} \in X^{\mathrm{nom}}$, and then we look for the most robust solution (i.e., optimize in the worst case) with tolerable degradations in the nominal quality. The tolerable degradations are given by $\xi \in \mathbb{R}^{k}$, whose component $\varepsilon_{i}$ represents the allowed degradation of $f_{i}^{\text {nom }}$.

For each $\hat{x} \in X^{\text {nom }}$, the point-based lightly robust problem with respect to (1) can be defined as follows:

$$
\begin{aligned}
\operatorname{minimize} & \left(\sup _{\xi \in \mathcal{U}} f_{1}(x, \xi), \ldots, \sup _{\xi \in \mathcal{U}} f_{k}(x, \xi)\right) \\
\text { subject to } & x \in X \\
& f_{i}^{\text {nom }}(x) \leq f_{i}^{\text {nom }}(\hat{x})+\varepsilon_{i} \text { for all } i=1, \ldots, k .
\end{aligned}
$$

In this formulation, we refer to $F^{\text {light }}(\hat{x}, \varepsilon):=\left\{x \in X: f_{i}^{\text {nom }}(x) \leq f_{i}^{\text {nom }}(\hat{x})+\right.$ $\varepsilon_{i}$ for all $\left.i=1, \ldots, k\right\}$ as the lightly robust feasible set. Lightly robust efficient solutions are identified by optimizing in the worst case with respect to the lightly robust feasible set. We say that a solution $x^{\text {light, } *} \in F^{\text {light }}(\hat{x}, \varepsilon)$ is lightly robust 


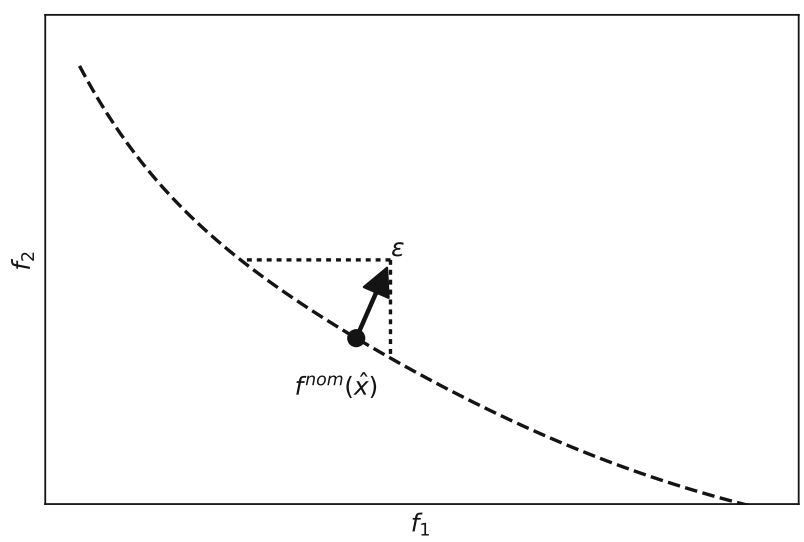

Fig. 1 Light robustness

efficient if there does not exists any solution $x^{\text {light, } * *} \in F^{\text {light }}(\hat{x}, \varepsilon)$ such that $f_{i}^{\mathrm{wc}}\left(x^{\text {light }, * *}\right) \leq f_{i}^{\mathrm{wc}}\left(x^{\text {light }, *}\right)$ for all $i=1, \ldots, k$ and $f_{j}^{\mathrm{wc}}\left(x^{\text {light }, * *}\right)<f_{j}^{\mathrm{wc}}\left(x^{\text {light }, *}\right)$ for at least one $j$. The set of lightly robust efficient solutions is denoted by $X^{\text {light, } \varepsilon}$.

By varying the value of $\varepsilon$, we can alter the trade-offs between robustness and the nominal quality in (5). Figure 1 illustrates the idea of light robustness with two objectives. The dashed line represents the set $f^{\text {nom }}\left(X^{\text {nom }}\right)$. The dot represents the nominal objective vector $f^{\text {nom }}(\hat{x})$ of the pre-selected nominal efficient solution $\hat{x}$. With a given $\varepsilon$, the area which can contain the nominal objective vectors of lightly robust efficient solutions is indicated by the dotted lines.

In Zhou-Kangas and Schöbel (2018), the gain in robustness and the price to be paid for robustness are defined for fixed nominal efficient and lightly robust efficient solutions. With fixed $\hat{x} \in X^{\text {nom }}$ and $x^{\text {light, } \varepsilon} \in X^{\text {light, },}$, we can quantify the gain in robustness by comparing their corresponding objective vectors in the worst case with the following measure:

$$
\operatorname{gain}\left(x^{\operatorname{light}, \varepsilon}, \hat{x}\right)=\left\|f^{\mathrm{wc}}(\hat{x})-f^{\mathrm{wc}}\left(x^{\operatorname{light}, \varepsilon}\right)\right\|_{\infty} .
$$

The notation $\|\cdot\|_{\infty}$ represents the $L_{\infty}$ norm, which tells the maximum gain in robustness among the objectives. Other norms can also be used, for example, $\|\cdot\|_{1}$ can give the value on the average gain in robustness among the objectives. Analogously, with fixed $\hat{x} \in X^{\text {nom }}$ and $x^{\text {light, } \varepsilon} \in X^{\text {light, } \varepsilon}$, we can quantify the price to be paid for robustness by comparing their corresponding objective vectors in the nominal scenario with the following measure:

$$
\operatorname{price}\left(x^{\operatorname{light}, \varepsilon}, \hat{x}\right)=\left\|f^{\text {nom }}(\hat{x})-f^{\text {nom }}\left(x^{\text {light }, \varepsilon}\right)\right\|_{\infty} .
$$

\subsection{Achievement scalarizing function}

As mentioned earlier, one approach to solving multiobjective optimization problems in the form of (3) is to formulate a single objective optimization subproblem by 
scalarizing. The achievement scalarizing function (Wierzbicki 1986) is one of the widely used scalarizing functions. In this paper, we utilize the following subproblem based on a variant of the achievement scalarizing function:

$$
\begin{array}{ll}
\text { minimize } & \alpha+\rho \sum_{i=1}^{k} w_{i}\left(f_{i}(x)-\bar{z}_{i}\right) \\
\text { subject to } & w_{i}\left(f_{i}(x)-\bar{z}_{i}\right) \leq \alpha \text { for all } i=1, \ldots, k \\
& x \in X
\end{array}
$$

where $\rho$ is a small scalar bounding the trade-offs among the objectives, $\bar{z}$ is a reference point and its components $\bar{z}_{i}$ are aspiration levels which represent the desired values of the objective function $f_{i}$ given by the decision maker. We have presented subproblem (8) in a differentiable form (assuming that the objective functions are differentiable), where the auxiliary variable $\alpha$ is used for the transformation from a minmax form (see e.g., Miettinen 1999). The positive weight vector $w$ sets a direction which the reference point is projected onto the set of objective vectors corresponding to the efficient solutions. We refer to $w$ as projection direction.

As discussed in the literature (e.g., in Branke et al. 2008; Miettinen 1999; Wierzbicki 1986), any optimal solution of (8) is an efficient solution for (2) and any efficient solution with trade-offs bounded by $\rho$ can be found by changing $\bar{z}$. Such solutions are also called properly efficient solutions (for details, see e.g., Wierzbicki 1986). The achievement scalarizing function works independently of the problem and preferences considered. For example, the reference point can be feasible or infeasible and the problem can be convex or nonconvex.

\section{Computing lightly robust efficient solutions}

\subsection{Lightly robust subproblem based on the achievement scalarizing function}

In this section, we assume that $\max _{\xi \in \mathcal{U}} f_{i}(x, \xi)$ exists for all $i=1, \ldots, k$ for a fixed $x \in X$. The existence of $\max _{\xi \in \mathcal{U}} f_{i}(x, \xi)$ for all $i=1, \ldots, k$ can be guaranteed for example when $X$ is finite, $\mathcal{U}$ is compact and $f_{i}(x, \cdot): \mathcal{U} \rightarrow \mathbb{R}^{k}$ is continuous in $\xi$ for every fixed $x \in X$. Based on (5) and (8), a lightly robust subproblem based on achievement scalarizing function can be given as follows:

$$
\begin{array}{ll}
\operatorname{minimize} & \alpha+\rho \sum_{i=1}^{k} w_{i}\left(\max _{\xi \in \mathcal{U}} f_{i}(x, \xi)-z_{i}\right) \\
\text { subject to } & f_{i}(x, \hat{\xi}) \leq f_{i}(\hat{x}, \hat{\xi})+\varepsilon_{i} \text { for all } i=1, \ldots, k \\
& w_{i}\left(\max _{\xi \in \mathcal{U}} f_{i}(x, \xi)-z_{i}\right) \leq \alpha \text { for all } i=1, \ldots, k \\
& x \in X .
\end{array}
$$

Theorem 1 Any optimal solution of (9) is a lightly robust efficient solution for (1). 
Proof Let $x^{*}$ be an optimal solution of (9) and assume that it is not lightly robust efficient to problem (1). Then there exists $x^{\prime} \in F^{\text {light }}(\hat{x}, \varepsilon)$, such that $f_{i}^{\text {wc }}\left(x^{\prime}\right) \leq$ $f_{i}^{\mathrm{wc}}\left(x^{*}\right)$ for all $i=1, \ldots, k$ and $f_{j}^{\mathrm{wc}}\left(x^{\prime}\right)<f_{j}^{\mathrm{wc}}\left(x^{*}\right)$ for at least one $j$. So we have $\max _{i}\left[w_{i}\left(\max _{\xi \in \mathcal{U}} f_{i}\left(x^{\prime}, \xi\right)-z_{i}\right)\right]+\rho \sum_{i=1}^{k}\left(\max _{\xi \in \mathcal{U}} f_{i}\left(x^{\prime}, \xi\right)-z_{i}\right)<\max _{i}\left[w_{i}\left(\max _{\xi \in \mathcal{U}} f_{i}\left(x^{*}, \xi\right)-\right.\right.$ $\left.\left.z_{i}\right)\right]+\rho \sum_{i=1}^{k}\left(\max _{\xi \in \mathcal{U}} f_{i}\left(x^{*}, \xi\right)-z_{i}\right)$. This contradicts with the assumption that $x^{*}$ is an optimal solution to (9). Thus $x^{*}$ is a lightly robust efficient solution to (1).

The formulation (9) involves finding the optimal solution of the maximization problem to identify the worst-case value of each objective function, i.e., the problem $\max _{\xi \in \mathcal{U}} f_{i}(x, \xi)$ for a fixed $x \in X$. So, using (9) to find a lightly robust solution requires solving a more complicated optimization problem than for a deterministic problem with (8). As mentioned in the literature (e.g., in Ben-Tal et al. 2009, 2015), problem (9) cannot be efficiently solved in a general case. Next, we reformulate the problem so that it can be efficiently solved by making some assumptions.

\subsection{Reformulating the lightly robust subproblem}

Reformulating problems to compute robust optimal solutions is a research topic with a long history in single objective optimization (see e.g., Ben-Tal and Nemirovski 1998; Bertsimas and Sim 2004; Soyster 1973).

Here we utilize the properties of $f_{i}(x, \xi)$ and $\mathcal{U}$. The following result has been presented in Corollary 2.14 in Tuy (2016):

Lemma 2 A real-valued quasi-convex function $g(x)$ on a compact convex set $C$ attains its maximum at an extreme point of $C$.

We consider the uncertainty set $\operatorname{con} v(\mathcal{U})$ which is called a polyhedral uncertainty set. It is defined by a set of scenarios $\mathcal{U}=\left\{\xi^{1}, \ldots, \xi^{m}\right\}$ as the extreme points of the convex hull. The result in Lemma 2 has been utilized in Kuhn et al. (2016) for reducing polyhedral uncertainty sets to discrete uncertainty sets involved in one of the objective functions in bi-objective optimization problems. It has also been utilized in Ehrgott et al. (2014) for replacing $\operatorname{con} v(\mathcal{U})$ by $\mathcal{U}$ for objective-wise uncertain problems. Since in this paper, we optimize in the point-based worst case for finding lightly robust efficient solutions, problem (9) has the following equivalent reformulation:

Theorem 3 Let conv $(\mathcal{U})$ be a polyhedral uncertainty set with extreme points $\mathcal{U}=$ $\left\{\xi^{1}, \ldots, \xi^{m}\right\}$ and $f_{i}$ quasi-convex in $\xi$ for every fixed $x \in X$ and $i=1, \ldots, k$. Problem (9) is equivalent to the following reformulation: 


$$
\begin{array}{ll}
\text { minimize } & \alpha+\rho \sum_{i=1}^{k} w_{i}\left(\gamma-\bar{z}_{i}\right) \\
\text { subject to } & x \in X \\
& f_{i}(x, \hat{\xi}) \leq f_{i}(\hat{x}, \hat{\xi})+\varepsilon \text { for all } i=1, \ldots, k \\
& w_{i}\left(\gamma-\bar{z}_{i}\right) \leq \alpha \\
& f_{i}\left(x, \xi^{j}\right) \leq \gamma \text { for all } i=1, \ldots, k \text { and } j=1, \ldots, m .
\end{array}
$$

Proof Based on Lemma 2, $f_{i}$ attains its maximum over conv $(\mathcal{U})$ at an extreme point of it with the assumption that $f_{i}$ is quasi-convex in $\xi$ for every fixed $x$. So we have

$$
\max _{\xi \in \operatorname{conv}(\mathcal{U})} f_{i}(x, \xi)=\max _{j} f_{i}\left(x, \xi^{j}\right)
$$

Corresponding to using $\alpha$ in (8), we use the auxiliary variable $\gamma$ (which is a scalar valued variable) to bound $\max _{i} \max _{j} f_{i}\left(x, \xi^{j}\right)$, i.e., we can solve (9) by considering the extreme points of $\operatorname{conv}(\mathcal{U})$ and the objective function which gives the largest value.

Based on e.g., Kuhn et al. (2016), polyhedral uncertainty sets include interval uncertainty sets (see e.g., Fischetti and Monaci 2009; Schöbel 2014) as special cases. When the uncertain parameters vary in intervals, we obtain a box as the uncertainty set, which is a special polyhedron. On the other hand, affine objective functions are also quasi-convex. So, (10) is also valid for problems with such characteristics.

If we have a multiobjective optimization problem with $\xi$ stemming from a set $\mathcal{U}$ with a finite number of scenarios, i.e., $\mathcal{U}$ is a finite uncertainty set, we can use the reformulation (10) to solve the problem. In this case, we do not need to assume that $f_{i}$ is quasi-convex in $\xi$ for every fixed $x \in X$ since we can directly compare the objective vectors in the scenarios.

\section{The LiRoMo method}

As introduced earlier, finding minmax robust efficient or nominal efficient solutions may not well serve the purpose of considering both robustness and the nominal quality. Minmax robust efficient solutions can have bad objective function values in other scenarios and the decision maker may not want to make decisions based on the worst possible realizations of the uncertain parameters. Nominal efficient solutions only concentrate on the nominal quality and other scenarios are ignored. Lightly robust efficient solutions can have both aspects incorporated. In this section, we propose the LiRoMo method to support the decision maker to find a most preferred lightly robust efficient solution with a preferred balance between robustness and the nominal quality. We first discuss the LiRoMo method in general. Then, we present its steps followed by a detailed discussion on the steps.

Using a reference point to find a most preferred efficient solution for the decision maker in an interactive method has been used in earlier research for deterministic problems for example in Nakayama and Sawaragi (1984), Wierzbicki (1986). In the LiRoMo method, we also utilize reference points as a means for the decision maker 
to express preference information. We first find a nominal efficient solution $\hat{x}$ which satisfies the preferences of the decision maker as well as possible. This is done by solving (8) based on the reference point specified by the decision maker. Then, based on the tolerable degradations of the nominal quality, which are also specified by the decision maker, we optimize in the worst case to find a lightly robust efficient solution by solving (9).

When the decision maker studies a lightly robust efficient solution, (s)he needs to understand its essence with respect to both robustness and the nominal quality. For the nominal quality, the decision maker needs to consider the nominal objective function values of the lightly robust efficient solution. For robustness, the concept of light robustness only finds the most robust solution with respect to the tolerable degradations. It is not enough to purely rely on this fact. Without additional information, it is hard for the decision maker to understand the trade-off between robustness and nominal quality, i.e., if the robustness gained is worthy of the sacrifice in the nominal quality.

Thus, we provide some additional information to help the decision maker to understand the trade-off between robustness and the nominal quality. In order to provide this information, we can utilize the gain in robustness to compare the lightly robust efficient and nominal efficient solutions in the worst case and use the price to be paid for robustness to compare the two solutions in the nominal scenario. As the objective function values can have very different scales, the original forms of gain in robustness and price to be paid for robustness presented in (6) and (7) need to be normalized. In the LiRoMo method, we calculate the gain in robustness as

$$
\operatorname{gain}\left(x^{\text {light }, \varepsilon}, \hat{x}\right)=\left\|\left(\frac{f_{1}^{\mathrm{wc}}\left(x^{\text {light }, \varepsilon}\right)-f_{1}^{\mathrm{wc}}(\hat{x})}{z_{1}^{\mathrm{nad}, \mathrm{wc}}-z_{1}^{\mathrm{uto}, \mathrm{wc}}}, \ldots, \frac{f_{k}^{\mathrm{wc}}\left(x^{\text {light }, \varepsilon}\right)-f_{k}^{\mathrm{wc}}(\hat{x})}{z_{k}^{\mathrm{nad}, \mathrm{wc}}-z_{k}^{\mathrm{uto}, \mathrm{wc}}}\right)^{T}\right\|_{\infty} .
$$

The value of $\operatorname{gain}\left(x^{\text {light, } \varepsilon}, \hat{x}\right)$ quantifies how much better the lightly robust efficient solution is in the worst case compared to the nominal efficient solution. The value of $\operatorname{gain}\left(x^{\text {light, } \varepsilon}, \hat{x}\right)$ represents the largest percentage that $x^{\text {light, } \varepsilon}$ is better in terms of the worst-case objective function values than $\hat{x}$ in the ranges of the objective vectors corresponding to the minmax robust efficient solutions. In addition, we also calculate the price to be paid for robustness:

$$
\begin{aligned}
& \operatorname{price}\left(x^{\text {light }, \varepsilon}, \hat{x}\right) \\
& =\left\|\left(\frac{f_{1}^{\text {nom }}\left(x^{\text {light }, \varepsilon}\right)-f_{1}^{\text {nom }}(\hat{x})}{z_{1}^{\text {nad }}-z_{1}^{\text {uto }}}, \ldots, \frac{f_{k}^{\text {nom }}\left(x^{\text {light }, \varepsilon}\right)-f_{k}^{\text {nom }}(\hat{x})}{z_{k}^{\text {nad }}-z_{k}^{\text {uto }}}\right)^{T}\right\|_{\infty} .
\end{aligned}
$$

The value of price $\left(x^{\operatorname{light}, \varepsilon}, \hat{x}\right)$ quantifies how much worse the lightly robust efficient solution is in the nominal scenario compared to the nominal efficient solution. The value of price $\left(x^{\operatorname{light}, \varepsilon}, \hat{x}\right)$ presents the largest percentage that $x^{\text {light, } \varepsilon}$ is worse than $\hat{x}$ in terms of the nominal objective function values in the ranges of the objective vectors corresponding to the nominal efficient solutions. If $\operatorname{price}\left(x^{\operatorname{light}, \varepsilon}, \hat{x}\right)$ is larger than 
$\operatorname{gain}\left(x^{\operatorname{light}, \varepsilon}, \hat{x}\right)$, more nominal quality is sacrificed compared to the gain in robustness. By combining the comparison of the two measures and the nominal objective vectors of the lightly robust efficient solution, the decision maker can consider her or his preferences in both respects and make informed decisions.

After understanding the presented lightly robust efficient solution, the decision maker needs to consider two different types of preference information for finding a more desired lightly robust efficient solution. First, (s)he needs to consider the preferences for a more interesting nominal efficient solution. Second, (s)he needs to consider the tolerable degradations in the nominal quality. The decision maker could also consider how much (s)he wants to gain in robustness by the maximum tolerable loss in the nominal quality which is specified by the tolerable degradations.

From the computation point of view, incorporating preferences on the gain in robustness can be achieved by adding additional constraints to the lightly robust problem. However, from a decision-making point of view, the decision maker should know the attainable objective function values and the trade-off between robustness and nominal quality very well. For example, with an unrealistic preference in the tolerance and gain, it may happen that there are no feasible lightly robust efficient solutions because the decision maker sacrifices too little but hopes to gain too much. This is why we do not request the preference information on the gain in robustness from the decision maker in LiRoMo but concentrate on finding a satisfactory lightly robust efficient solution by allowing the decision maker to alter her or his preferences in the nominal objective vector in the form of reference points and specifying the tolerable degradations.

The steps of the LiRoMo method are the followings:

Initialization. Present $z^{\text {ideal }}, z^{\text {nad }}$ and $z^{\text {ideal,wc }}$ and $z^{\text {nad,wc }}$ to the decision maker. Set the iteration counter $c=0$.

Step 1. Ask the decision maker to specify the desired values of each objective function which forms the reference point $\bar{z}$.

Step 2. Solve (8) to find a nominal efficient solution $\hat{x}^{c}$.

Step 3. Present the objective vector corresponding to $\hat{x}^{c}$ to the decision maker.

Step 4. Ask the decision maker to specify her or his preferences on how much (s)he is willing to sacrifice in the nominal quality to gain robustness which forms the tolerable degradations $\varepsilon$ for the preferred nominal solution.

Step 5. With $\hat{x}^{c}$ and $\varepsilon$, solve (9) to find a lightly robust efficient solution $x^{(\text {light }, \varepsilon)_{c}}$ and compute the gain in robustness gain $\left(x^{(\text {light }, \varepsilon)_{c}}, \hat{x}^{c}\right)$ and the price to be paid for robustness price $\left(x^{(\mathrm{light}, \varepsilon)_{c}}, \hat{x}^{c}\right)$.

Step 6. Present the nominal objective vectors corresponding to $x^{(\text {light }, \varepsilon)_{c}}$ and $\hat{x}^{c}$ together with the values of $\operatorname{gain}\left(x^{(\mathrm{light}, \varepsilon)_{c}}, \hat{x}^{c}\right)$ and price $\left(x^{(\mathrm{light}, \varepsilon)_{c}}\right.$, $\hat{x}^{c}$ ) to the decision maker.

Step 7. If the decision maker is satisfied, terminate the solution process and set $x^{(\text {light, } \varepsilon)_{c}}$ as the final solution. Otherwise, continue.

Step 8. If the decision maker wishes to alter the trade-offs between robustness and the nominal quality, i.e., modify the tolerable degradations, ask the decision maker to give a new preferred value of $\varepsilon$, set $c=c+1$ and go to step 3. If the decision maker wants to change her or his 
preferences on the nominal efficient solution, set $c=c+1$ and go to step 1 .

In the initialization step, the nominal ideal and nadir objective vectors are presented to the decision maker to help her or him to have a general idea of the ranges of the objective function values among the nominal solutions. This helps the decision maker to specify the reference point in step 1 . The robust ideal objective vector and the robust nadir objective vector are also presented to help the decision maker to grasp a picture on the ranges of the objective function values of solutions in the worst case if (s)he fully concentrates on robustness. This can help her or him to specify the tolerable degradations in the nominal quality later. In step 2, we solve (8) to find the nominal efficient solution $\hat{x}^{c}$ using $\bar{z}$ as the reference point. Then, we can present the objective vector corresponding to $\hat{x}^{c}$ to the decision maker in step 3 and ask the decision maker to specify her or his preferences on $\varepsilon$ in step 4 .

In step 5, with $\hat{x}^{c}$ and $\varepsilon$, we solve (9) to find a lightly robust efficient solution $x^{(\text {light, } \varepsilon)_{c}}$. The value of $\varepsilon$ is closely related to the reservation levels (see e.g., Granat et al. 1994). Reservation levels are limits of objective function values that the decision maker cannot accept to go beyond. In our context, the decision maker wants to avoid any nominal objective vector worse than $f^{\text {nom }}(\hat{x})+\varepsilon$. So, with a fixed $f^{\text {nom }}(\hat{x})$, if a decision maker changes the value of $\varepsilon$, (s)he changes the limits of the acceptable nominal objective vector beyond which the corresponding solutions should be avoided. This means that changing the value of $\varepsilon$ can be understood as changing the reservation levels.

When solving (9), we use $f^{\text {nom }}\left(\hat{x}^{c}\right)+\varepsilon$ as the reference point. This is because of two reasons. First, the value of $f^{\text {nom }}\left(\hat{x}^{c}\right)+\varepsilon$ is still acceptable for the decision maker. Second, (s)he is expecting the most robust solution within the range of $f^{\text {nom }}\left(\hat{x}^{c}\right)$ and $f^{\text {nom }}\left(\hat{x}^{c}\right)+\varepsilon$, so (s)he is willing to sacrifice until the limits of the acceptable nominal objective function values to gain robustness. We set the projection vector $w=\frac{1}{f^{\mathrm{nom}}\left(\hat{x}^{c}\right)+\varepsilon-f^{\mathrm{nom}}\left(\hat{x}^{c}\right)}=\frac{1}{\varepsilon}$ because in the ideal situation, $\hat{x}^{c}$ is a lightly robust efficient solution. If $\hat{x}^{c}$ is not a lightly robust efficient solution, we may preserve the preferences on the trade-offs among the objectives with this projection direction.

In order to efficiently solve (9), we can use the reformulation presented in the subproblem (10). Correspondingly, we can compare the objective function values in the scenarios which specify $\mathcal{U}$ to find the point-based worst-case objective vector for computing the value of $\operatorname{gain}\left(x^{(\operatorname{light}, \varepsilon)_{c}}, \hat{x}^{c}\right)$. For computing the value of price $\left(x^{(\operatorname{light}, \varepsilon)_{c}}, \hat{x}^{c}\right)$, we only need to evaluate the two solutions in the nominal scenario. If the problem does not meet the assumptions in the reformulation, it is possible to represent the uncertainty set by a set of discrete scenarios by utilizing a good sampling technique presented e.g., in Simpson et al. (2001). When we replace the uncertainty set by a set of sampled discrete scenarios, we can use the reformulation presented in (10).

In step 6 , we visualize $f^{\text {nom }}\left(x^{(\text {light }, \varepsilon)_{c}}\right)$ and $f^{\text {nom }}\left(\hat{x}^{c}\right)$ together with the values of $\operatorname{gain}\left(x^{(\operatorname{light}, \varepsilon)_{c}}, \hat{x}^{c}\right)$ and price $\left(x^{(\operatorname{light}, \varepsilon)_{c}}, \hat{x}^{c}\right)$ to the decision maker. As mentioned earlier, by combining the two types of information, the decision maker can understand the trade-offs in the objectives and also consider balancing between robustness and the nominal quality. 


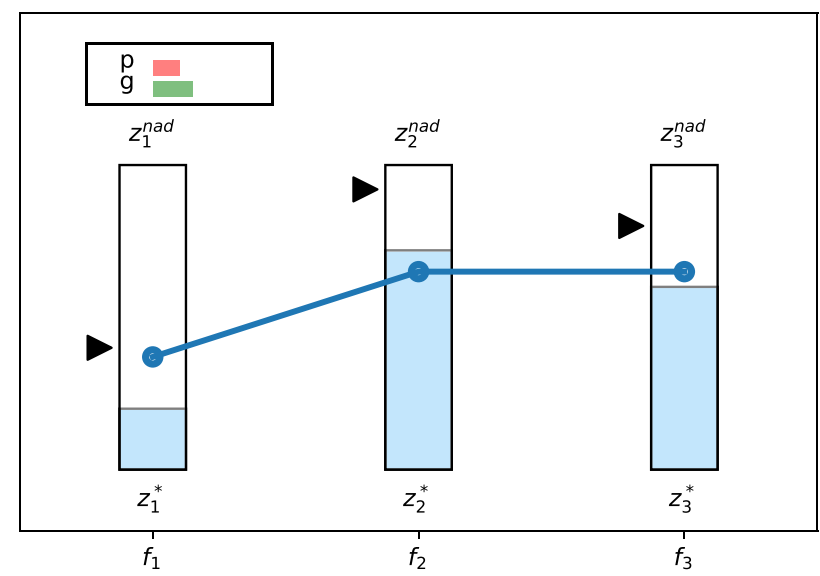

Fig. 2 Visualizing a lightly robust efficient solutions

For illustrating the computed lightly robust efficient solutions in step 6, we augment the value path visualization (Miettinen 2014) to help the decision maker to understand the essence of the solutions. Figure 2 shows an example of visualizing a lightly robust efficient solution with its corresponding nominal objective vector and other related information. In the example figure, there are three objectives represented by bars. The minima and maxima of the bars represent the nominal ideal and nadir values, respectively. The filled part of the bar is the objective function value of the nominal efficient solution which satisfies the reference point best. The triangles mark the tolerable degradations (i.e., the value until which the decision maker is willing to sacrifice to gain robustness). The value path illustrates the nominal objective vector of the current lightly robust efficient solution. The gain in robustness (marked as $\mathrm{g}$ in the figure) and the price to be paid for robustness (marked as $\mathrm{p}$ in the figure) are illustrated by the horizontal bars on the upper left corner.

When a decision maker sees the visualization, (s)he considers the nominal objective function values of the lightly robust efficient solution. This is because of the nature of concentration on the nominal scenario in light robustness. If the values are acceptable, (s)he then further considers if the gained robustness is worthy of the sacrifice in the nominal quality by simply comparing the lengths of the bars marked by $g$ and $\mathrm{p}$. If the decision maker asks, we can present their numerical values.

To summarize, in the figure, the decision maker can see five types of information:

- The nominal objective function values of the current nominal efficient solution which satisfies the reference point best in the colored bars.

- The nominal objective function values of the current lightly robust efficient solution.

- The change in the nominal quality, i.e., the difference between the markers of the value path and the filled part of the bars.

- How much better the current lightly robust efficient solution is compared to the worst acceptable one. 
- How much robustness (s)he has gained in the solution compared to the sacrificed nominal quality.

The five types of information together help the decision maker to understand the current lightly robust efficient solution in terms of its nominal quality, the relationships with the nominal efficient solution and the trade-offs between robustness and nominal quality. They also help the decision maker to analyze what kind of changes (s)he should make to get a more desired solution by specifying the preferences for the next iteration. If (s)he sees that the tolerable degradations can be still modified while the gain in robustness is not sufficient, (s)he can relax the value of $\varepsilon$ to get a more robust solution. If (s)he is not willing to sacrifice more in the nominal quality or is not satisfied by the nominal objective function values of the lightly robust efficient solution, (s)he can try to provide a new reference point to change the nominal efficient solution.

After presenting the lightly robust efficient solution, if the decision maker is satisfied, we terminate the solution process with $x^{(\text {light, } \varepsilon)_{c}}$ as the final solution. If the decision maker wishes to continue the solution process, we calculate a new solution based on her or his preferences. By interacting with the decision maker, we support her or him to find a satisfactory lightly robust efficient solution based on her or his preferences on the nominal objective function values and the tolerable degradations. If the decision maker so desires, the objective vector of the final solution in the worst case can also be presented. In case that the worst-case objective vector is not acceptable, the decision maker can provide new preferences to get a new solution.

\section{Example in investment portfolio optimization}

Portfolio optimization problems have been considered in the literature but with different concepts of robustness. In this section, we formulate a simple investment portfolio optimization problem with uncertainty in future developments. We solve this problem to demonstrate the ability of the LiRoMo method in supporting the decision maker to find a most preferred balance between robustness and the nominal quality.

The main products of a start-up Company A are software products. Now the owners of the company are considering investing in some stocks for long-term return. Just like in any portfolio investment problem, they want to maximize the return on investment and minimize the risks. They plan to study the long-term historical data of the stocks to find a good composition for their investment portfolio. However, it is also possible that their investment will be withdrawn as a mid-term or even a short-term investment if they discover and initiate a new interesting project. Here, the uncertainty comes from the fact that Company A does not know exactly which of the time frames should be used in the portfolio optimization. In this case, we have three discrete scenarios in the uncertainty set including the short-term data, mid-term data, and long-term data. Company A wants to concentrate on a long-term investment, so using long-term data is considered as the nominal scenario and using short- and mid-term data are considered as the other two possible scenarios.

In the solution process, we aim at finding a composition of investments, which is good with respect to all scenarios and especially not too bad as a long-term investment. 
We apply the LiRoMo method and interact with a decision maker to find a lightly robust investment portfolio with good return and acceptable risks in long-term but at the same time with not too bad return and risks at any earlier withdrawal.

As mentioned before, the objectives of the investment include maximizing the return on investment and minimizing the risks. Different risk measures providing different insights are used in the literature such as the standard deviation, $\beta$ index, Sharpe index, and Treynor index (see e.g., Reilly and Brown 2011). In this paper, we use the Sharpe index and the Treynor index to be maximized as the standard deviation is related to the Sharpe index and the $\beta$ index is related to the Treynor index and we only consider indices with a low correlation. Briefly speaking, the Sharpe index indicates how well a portfolio uses risk to get return and the Treynor index measures the volatility in the market to calculate the value of a portfolio adjusted risk. In order to avoid any failure in unexpected behaviors in a single or a few good stocks, we also minimize the maximum amount of investment in a single stock among all the invested stocks. The problem is formulated as a multiobjective optimization problem with uncertain parameters in the objectives:

$$
\begin{array}{ll}
\operatorname{maximize} & f_{1}(x)=\sum_{i=1}^{n} \frac{p_{i j}^{t}-p_{i j}^{t-1}}{p_{i j}^{t-1} x_{i}} \\
\text { maximize } & f_{2}(x)=\frac{1}{\sigma_{j}}\left(\sum_{i=1}^{n} \frac{p_{i j}^{t}-p_{i j}^{t-1}}{p_{i j}^{t-1}} x_{i}-\tilde{r}\right) \\
\text { maximize } & f_{3}(x)=\frac{1}{\beta_{j}}\left(\bar{r}_{j}-\tilde{r}\right) \\
\text { minimize } & f_{4}(x)=\max _{i} x_{i} \\
\text { subject to } & x_{i} \geq 0 \\
& \sum_{i=1}^{n} x_{i}=1 \\
& p_{i j}^{t} \in P_{i}^{t} \\
& p_{i j}^{t-1} \in P_{i}^{t-1} .
\end{array}
$$

In the formulation, $f_{1}$ represents the return on investment, $f_{2}$ represents the Sharpe index, $f_{3}$ represents the Treynor index, and $f_{4}$ represents the maximum investment in a single stock. In this formulation, if the value of $f_{1}$ is smaller than 1 , there is loss in the investments. The decision variables $x_{i}$ represent the proportion of the total amount of investment in the stock $i$ and there are in total $n$ stocks for investment. The parameters $p_{i j}^{t}, p_{i j}^{t-1}$ are the historical buying and selling prices of the stock $i$ when the time frame $j$ is used, where $t$ stands for the most recent time and $t-1$ represent the previous time period. The notation $\tilde{r}$ represents the risk-free rate and $\bar{r}_{j}$ is the average return rate of the portfolio when the time frame $j$ is used. The standard deviation of the return on the portfolio in the time frame $j$ is denoted by $\sigma_{j}$. The beta index is denoted by $\beta_{j}$ in the time frame $j$. The value of the beta index depends on the return on investment of the investment portfolio.

The parameters can have different values depending on the time frame used. Each parameter has three different possible values to consider, i.e., when short-, mid-, and long-term data are used in optimizing the portfolio. So, we have two uncertainty 
sets $P_{i}^{t}$ and $P_{i}^{t-1}$ and each uncertainty set has three elements. For example, we have $P_{i}^{t}=\left\{p_{i s}^{t}, p_{i m}^{t}, p_{i l}^{t}\right\}$ where $s$ stands for the short-time frame, $m$ stands for the mid-term time frame and $l$ stands for long-time frame. The nominal values of the sets are $p_{i l}^{t}$ and $p_{i l}^{t-1}$. The indices $\sigma_{j}$, and $\beta_{j}$ depend on the data in the time frame (i.e., $p_{i j}^{t-1}$ and $p_{i j}^{t}$ ). Note that in the formulation, $f_{4}(x)$ does not involve uncertain parameters but different investment portfolios (i.e., solutions) correspond to different values of $f_{4}(x)$. Problem (13) can be reformulated for computing lightly robust efficient solutions based on problem (5).

As for the historical data of the portfolios, we downloaded 10 different NASDAQ stocks from Google finance https://www.google.com/finance and computed the needed indices. We started our solution process by calculating the nominal ideal objective vector and approximating the nadir objective vector with the payoff table. We have $z^{\text {ideal }}=(1.677,118.85,0.1266,0.1)^{T}$ and $z^{\text {nad }}=(0.636,25.665,0.0478,1)^{T}$. We also calculated and approximated the robust ideal and nadir objective vectors $z^{\text {ideal, } \mathrm{wc}}=(1.34,89.67,0.093,0.1)^{T}$ and $z^{\text {nad, } w c}=(0.38,23.37,0.0289,1)^{T}$. One should note that since the first three objectives are to be maximized, the corresponding components in the ideal objective vector are higher than those of the nadir objective vector. In this problem, the uncertain parameters stem from discrete sets, so we utilized the reformulation (10) to compute the lightly robust efficient solutions.

Initialization. We presented the nominal ideal and nadir objective vectors to the decision maker as the ranges of the objective function values of the nominal efficient solutions. We also presented the robust ideal and nadir objective vectors to the decision maker as the ranges in the minmax robust efficient solutions.

Initial solution. Based on the ranges, the decision maker specified the reference point: $\bar{z}^{0}=(1.1,75,0.1,0.5)^{T}$. With the preferences, we first solved (8) and found $\hat{x}^{0}$. Then, we presented the nominal objective function values of $\hat{x}^{0}$ to the decision maker and asked him to specify his preferences on the tolerable degradation. Then, we solved the reformulated lightly robust problem and found an initial lightly robust efficient solution and presented it to the decision maker as shown in Fig. 3. Based on this solution, the decision maker could not accept the loss in the investments even though the price to be paid for robustness resulted in a good gain in robustness.

Iteration 1. So, we asked him to specify a new reference point. Based on the new reference point $\bar{z}^{1}=(1.1,85,0.1,0.35)^{T}$, we calculated $\hat{x}^{1}$. Based on the objective function values of $\hat{x}^{1}$, the decision maker specified the tolerable degradations. With the tolerable degradations $\epsilon^{1}=(0.05,10,0.008,0.09)^{T}$ and $\hat{x}^{1}$, we calculated a new lightly robust efficient solution by solving (10). We presented its nominal objective vector to the decision maker as in Fig. 4. With the solution, the decision maker could make only little profit even though he noticed that the price to be paid for robustness resulted in a much bigger amount of gain in robustness.

Iteration 2. The decision maker decided to try to provide a really good aspiration level to the return on investment with less emphasis on other objectives. With $\bar{z}^{2}=$ $(1.8,20,0.05,1)^{T}$, we calculated $\hat{x}^{2}$. Based on $f^{\text {nom }}\left(\hat{x}^{2}\right)$, the decision maker specified $\epsilon^{2}=(0.1,28,0,0.1)^{T}$, we again solved (10) and found a new lightly robust efficient solution as illustrated in Fig. 5. The decision maker noticed that even though the 


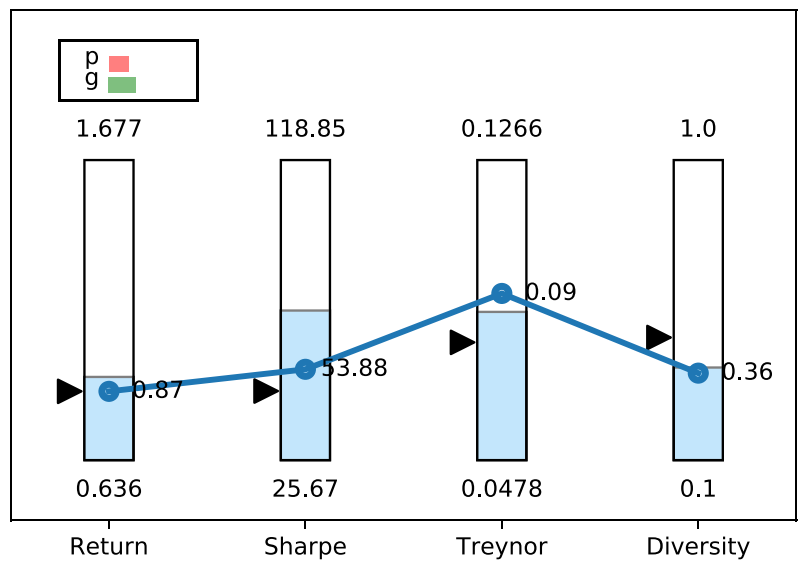

Fig. 3 Initial solution

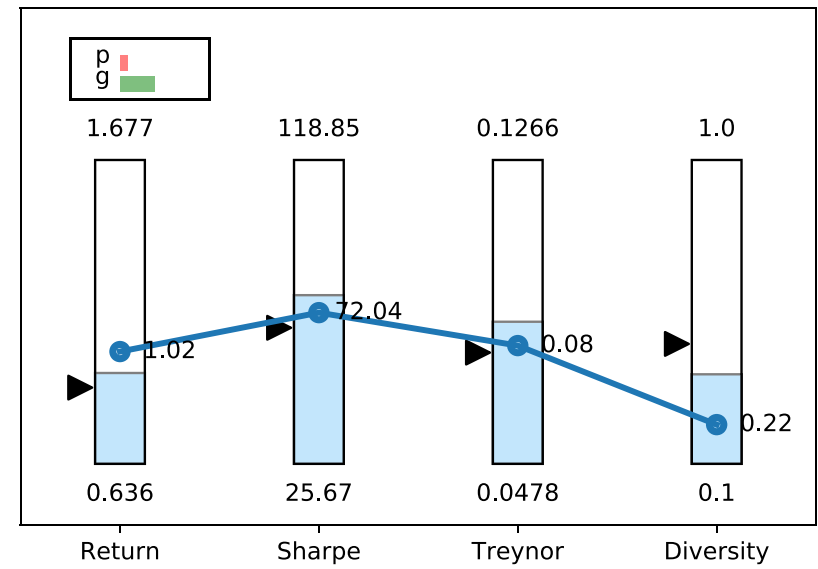

Fig. 4 Iteration 1

return on investment was good, the investment was rather risky and the lightly robust efficient solution was very different from the nominal efficient solution. Since the nominal objective function values of the lightly robust efficient solution were not acceptable, he did not even consider the trade-off between robustness and the nominal quality.

Iteration 3. The decision maker provided a new reference point $\bar{z}^{3}=(1.2,40,0.11,1)^{T}$ with which we computed $\hat{x}^{3}$. With the presented $f^{\text {nom }}\left(\hat{x}^{3}\right)$, the decision maker specified the tolerable degradations $\epsilon^{3}=(0.08,20,0.008,0.09)^{T}$. Based on $\hat{x}^{3}$ and $\epsilon^{3}$, we calculated and presented a new lightly robust efficient solution to the decision maker as in Fig. 6. In this solution, the decision maker noticed that the Treynor index increased as she intended. However, there were losses in the investment. As before, since this solution was not acceptable, he did not consider comparing the values of gain in robustness and price to be paid for robustness. 


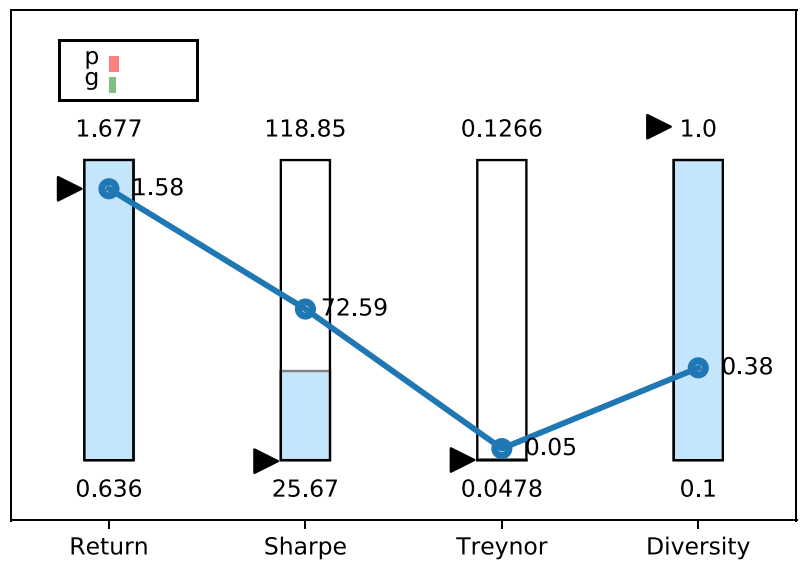

Fig. 5 Iteration 2

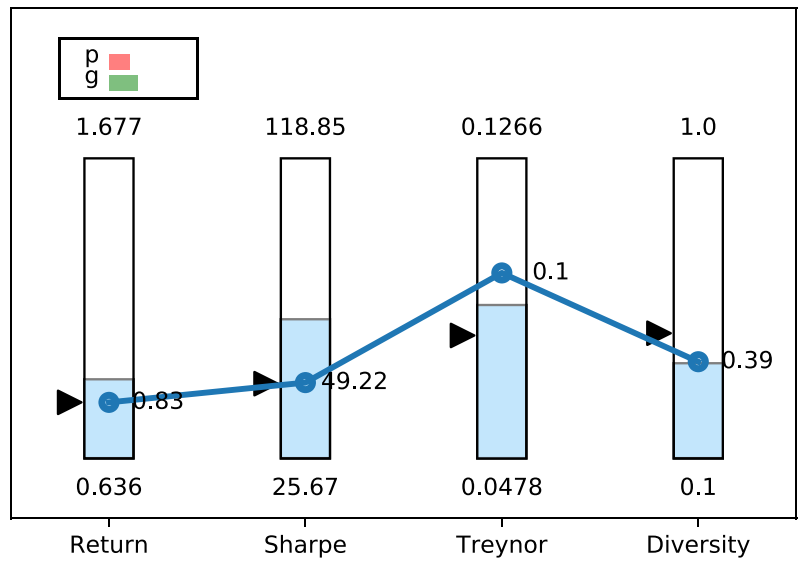

Fig. 6 Iteration 3

Iteration 4. The decision maker tried with another reference point with a better aspiration level on return on investment $\bar{z}^{4}=(1.5,85,0.1,0.35)^{T}$. After knowing $f^{\text {nom }}\left(\hat{x}^{4}\right)$ which we computed, he also decided not to allow the return on investment and the Sharpe index to degrade as much as in previous iterations with $\epsilon^{4}=(0.05,10,0.008,0.09)^{T}$. Using $\hat{x}^{4}$ and $\epsilon^{4}$, we found a new lightly robust efficient solution as in Fig. 7. The decision maker liked the solution in terms of its nominal objective function values and the balance in the price to be paid for and the gain in robustness.

Termination. Even though the decision maker liked the solution in the previous iteration, he still provided a new reference point $\bar{z}^{5}=(1.7,55,0.1,0.25)^{T}$ to try to increase the return on investment by lowering the Sharpe index. After knowing $f^{\text {nom }}\left(\hat{x}^{5}\right)$, he decided to further reduce the tolerable degradations of the return on investment and the Sharpe index with $\epsilon^{5}=(0.04,5,0.008,0.09)^{T}$. With the new preference infor- 


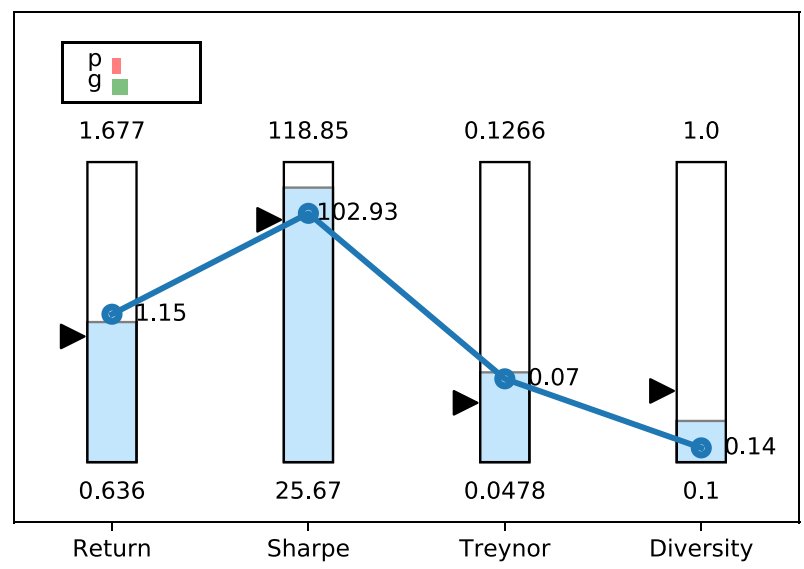

Fig. 7 Iteration 4

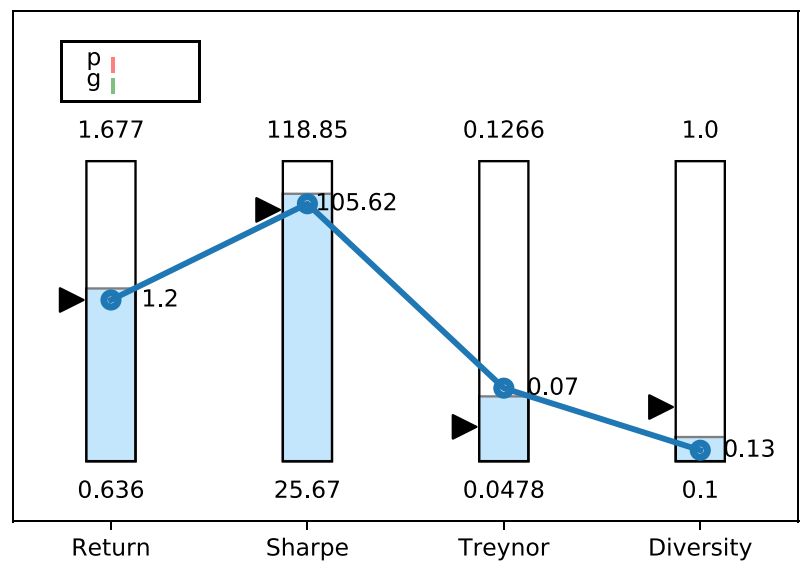

Fig. 8 Final solution

mation, the solution present in Fig. 8 was obtained. The decision maker found that this lightly robust efficient solution had better nominal objective function values than in the previous iteration with the reduced tolerable degradations. In addition, the gain in robustness was worthy of the sacrifice in the nominal quality with a preferred balance. So, he decided to terminate the solution process.

During the solution process, the decision maker chose to provide reference points and tolerable degradations for the computation of lightly robust efficient solutions. In the beginning of the solution process, the decision maker took some iterations to learn about the attainable objective function values and how he could utilize the reference point and the value of $\varepsilon$ to guide the solution process toward the kind of solutions she desires. In the last two iterations, the decision maker was able to find a desirable lightly robust efficient solution and utilize $\varepsilon$ to fine-tune the solution. In addition, the comparison between the price to be paid for and the gain in robustness served as useful 
information for the decision maker to make an informed decision. The final solution found was satisfactory with a good return on investment and acceptable risk levels.

\section{Conclusions}

In this paper, we considered multiobjective optimization problems where some parameters are uncertain in the objective functions. In order to support the decision maker to find a most preferred solution with a good balance between robustness and the nominal quality, we developed the LiRoMo method by utilizing the concept of light robustness. It is the first interactive method using light robustness. In the LiRoMo method, the decision maker can alter the trade-offs between robustness and the nominal quality by changing tolerable degradations in the nominal quality. As a support for the decision maker to consider the balance, we quantified the price to be paid for robustness and the gain in robustness in each computed lightly robust efficient solution. In addition, we visualized the lightly robust efficient solutions and related information to help the decision maker to understand them with an augmented value path visualization.

We formulated an investment portfolio optimization problem and solved it with the LiRoMo method involving a decision maker to demonstrate the advantages of the method. With the support provided by the method, the decision maker was able to explore the objective function values of the solutions of the problem and eventually found a lightly robust efficient solution with a good balance between robustness and the nominal quality.

In this paper, we reformulated the lightly robust problem based on the achievement scalarizing function under some assumptions: the objective functions are quasi-convex with respect to the uncertain parameter with a fixed decision vector and the uncertain parameters stem from polyhedral uncertainty sets. An immediate continuation of this research is to efficiently compute lightly robust solutions for more general problems. In the current version of the interactive method, it is possible that the trade-offs between the objective function values of the lightly robust efficient solutions are different from those of the nominal efficient solution. The reason is that the preferences on the nominal objective function values are first considered in the form of a reference point. When calculating the lightly robust efficient solution, the focus is on the robustness. Thus, another interesting future research direction is to refine interactive methods to further maintain the preferences set in the nominal objective function values in the lightly robust efficient solutions.

Acknowledgements Open access funding provided by University of Jyväskylä (JYU). We thank Dr. Dmitry Podkopaev for various discussions in formulating the investment portfolio optimization problem.

Open Access This article is distributed under the terms of the Creative Commons Attribution 4.0 International License (http://creativecommons.org/licenses/by/4.0/), which permits unrestricted use, distribution, and reproduction in any medium, provided you give appropriate credit to the original author(s) and the source, provide a link to the Creative Commons license, and indicate if changes were made. 


\section{References}

Ben-Tal A, Nemirovski A (1998) Robust convex optimization. Math Oper Res 23(4):769-805. https://doi. org/10.1287/moor.23.4.769

Ben-Tal A, Goryashko A, Guslitzer E, Nemirovski A (2004) Adjustable robust solutions of uncertain linear programs. Math Progr 99(2):351-376. https://doi.org/10.1007/s10107-003-0454-y

Ben-Tal A, El Ghaoui L, Nemirovski A (2009) Robust optimization. Princeton University Press, Princeton

Ben-Tal A, Bertsimas D, Brown DB (2010) A soft robust model for optimization under ambiguity. Oper Res 58(4-part-2):1220-1234. https://doi.org/10.1287/opre.1100.0821

Ben-Tal A, Hazan E, Koren T, Mannor S (2015) Oracle-based robust optimization via online learning. Oper Res 63(3):628-638. https://doi.org/10.1287/opre.2015.1374

Bertsimas D, Sim M (2004) The price of robustness. Oper Res 52(1):35-53. https://doi.org/10.1287/opre. 1030.0065

Bitran GR (1980) Linear multiple objective problems with interval coefficients. Manag Sci 26(7):694-706. https://doi.org/10.1287/mnsc.26.7.694

Bokrantz R, Fredriksson A (2017) Necessary and sufficient conditions for Pareto efficiency in robust multiobjective optimization. Eur J Oper Res 262(2):682-692. https://doi.org/10.1016/j.ejor.2017.04.012

Branke J, Deb K, Miettinen K, Slowinski R (eds) (2008) Multiobjective optimization, interactive and evolutionary approaches. Springer, New York

Deb K, Miettinen K, Chaudhuri S (2010) Toward an estimation of nadir objective vector using a hybrid of evolutionary and local search approaches. IEEE Trans Evol Comput 14(6):821-841. https://doi.org/ 10.1109/TEVC.2010.2041667

Dranichak GM, Wiecek MM (2018) On highly robust efficient solutions to uncertain multiobjective linear programs. Eur J Oper Res. https://doi.org/10.1016/j.ejor.2018.07.035

Ehrgott M, Ide J, Schöbel A (2014) Minmax robustness for multi-objective optimization problems. Eur J Oper Res 239(1):17-31. https://doi.org/10.1016/j.ejor.2014.03.013

Eichfelder G, Krüger C, Schöbel A (2017) Decision uncertainty in multiobjective optimization. J Glob Optim 69(2):485-510. https://doi.org/10.1007/s10898-017-0518-9

Fischetti M, Monaci M (2009) Light robustness. In R. K. Ahuja, R. H. Möhring, and C. D. Zaroliagis, editors, Robust and Online Large-Scale Optimization: Models and Techniques for Transportation Systems, pages 61-84. Springer. https://doi.org/10.1007/978-3-642-05465-5_3

Fliege J, Werner R (2014) Robust multiobjective optimization \& applications in portfolio optimization. Eur J Oper Res 234(2):422-433. https://doi.org/10.1016/j.ejor.2013.10.028

Goberna M, Jeyakumar V, Li G, Vicente-Pérez J (2018) Guaranteeing highly robust weakly efficient solutions for uncertain multi-objective convex programs. Eur J Oper Res 270(1):40-50. https://doi.org/ 10.1016/j.ejor.2018.03.018

Goerigk M, Schöbel A (2016) Algorithm engineering in robust optimization. In: Kliemann L, Sanders P (eds) Algorithm engineering: selected results and surveys. Springer, New York, pp 245-279. https:// doi.org/10.1007/978-3-319-49487-6_8

Granat J, Kreglewski K, Paczynski J, Stachurski A (1994) IAC-DIDASN++ modular modeling and optimization systems theoretical foundations. Report of the Institute of Automatic. Control, Warsaw University of Technology

Gutjahr WJ, Pichler A (2016) Stochastic multi-objective optimization: a survey on non-scalarizing methods. Ann Oper Res 236(2):475-499. https://doi.org/10.1007/s10479-013-1369-5

Hassanzadeh F, Nemati H, Sun M (2014) Robust optimization for interactive multiobjective programming with imprecise information applied to R\&D project portfolio selection. Eur J Oper Res 238(1):41-53. https://doi.org/10.1016/j.ejor.2014.03.023

Ide J, Schöbel A (2016) Robustness for uncertain multi-objective optimization: a survey and analysis of different concepts. OR Spectr 38(1):235-271. https://doi.org/10.1007/s00291-015-0418-7

Inuiguchi M, Ichihashi H, Tanaka H (1990) Fuzzy programming: a survey of recent developments. In Shi-Yu, $\mathrm{H}$, Jaques $\mathrm{T}$ (eds) Stochastic versus fuzzy approaches to multiobjective mathematical programming under uncertainty. Springer, New York, pp 45-68. https://doi.org/10.1007/978-94-009-2111-5_4

Kuhn K, Raith A, Schmidt M, Schöbel A (2016) Bi-objective robust optimisation. Eur J Oper Res 252(2):418-431. https://doi.org/10.1016/j.ejor.2016.01.015

Kuroiwa D, Lee GM (2012) On robust multiobjective optimization. Vietnam J Math 40(2-3):305-317

Miettinen K (1999) Nonlinear multiobjective optimization. Kluwer Academic Publishers, Berlin 
Miettinen K (2014) Survey of methods to visualize alternatives in multiple criteria decision making problems. OR Spectrum 36(1):3-37. https://doi.org/10.1007/s00291-012-0297-0

Nakayama H, Sawaragi Y (1984) Satisficing trade-off method for multiobjective programming and its applications. IFAC Proc Vol 17(2):1345-1350. https://doi.org/10.1016/S1474-6670(17)61162-5

Reilly FK, Brown KC (2011) Investment analysis and portfolio management. Cengage Learning, South Western

Schöbel A (2014) Generalized light robustness and the trade-off between robustness and nominal quality. Math Methods Oper Res 80(2):161-191. https://doi.org/10.1007/s00186-014-0474-9

Simpson TW, Lin DK, Chen W (2001) Sampling strategies for computer experiments: design and analysis. Int J Reliab Appl 2(3):209-240

Soyster AL (1973) Technical note-convex programming with set-inclusive constraints and applications to inexact linear programming. Oper Res 21(5):1154-1157. https://doi.org/10.1287/opre.21.5.1154

Steuer RE (1986) Multiple criteria optimization: theory, computation, and applications. Wiley, Hoboken

Steuer RE, Choo E-U (1983) An interactive weighted Tchebycheff procedure for multiple objective programming. Math Progr 26(3):326-344. https://doi.org/10.1007/BF02591870

Tuy H (2016) Convex analysis and global optimization. Springer, New York

Wiecek MM, Dranichak GM (2016) Robust multiobjective optimization for decision making under uncertainty and conflict. In: Gupta A, Capponi A, Smith JC, Greenberg HJ (eds) Optimization challenges in complex, networked and risky systems. INFORMS, pp 84-114. https://doi.org/10.1287/educ.2016. 0153

Wierzbicki AP (1986) On the completeness and constructiveness of parametric characterizations to vector optimization problems. OR Spectrum 8(2):73-87. https://doi.org/10.1007/BF01719738

Zhou-Kangas Y, Schöbel A (2018) The price of multiobjective robustness: analyzing solution sets to uncertain multiobjective problems (Manuscript) 